\title{
Traumatic Cervical Internal Carotid Artery Occlusion in an Infant
}

\author{
-Case Report- \\ Takahiro IgARASHI, Tatsuro MORI, Nobuhiro MORO, Hideki OsHIMA,
and Yoichi KATAYAMA
}

Department of Neurological Surgery, Nihon University School of Medicine, Tokyo

\begin{abstract}
A 10-month-old boy presented with traumatic internal carotid artery (ICA) occlusion caused by blunt injury after falling from a baby carrier attached to a standing bicycle. Physical examination found bruises on the scalp in the right temporal region and the right shoulder, but no wound in the neck. Chest radiography showed a right clavicular fracture. He developed left hemiparesis at 19 hours after the injury. Computed tomography revealed cerebral infarct and angiography showed cervical ICA occlusion. Conservative therapy with hemodilution was given under a diagnosis of cervical ICA occlusion caused by extension and rotational head injury. Traumatic cervical ICA occlusion due to blunt injury is not uncommon in adults, but extremely rare in infants. We would like to emphasize the dangers of leaving a baby unattended in a carrier attached to a standing bicycle.
\end{abstract}

Key words: internal carotid artery occlusion, blunt injury, infant, dissection, cerebral infarction

\section{Introduction}

Traumatic internal carotid artery (ICA) occlusion may result from either penetrating or blunt injury. Blunt injury includes both direct hit to the neck and extension or rotational injury. The ICA occlusion may be located in either the extracranial or intracranial ICA. Traumatic ICA occlusion presents with delayed onset of symptoms and is associated with high mortality and morbidity. The first case of traumatic ICA occlusion caused by blunt injury to the neck was reported in 1872.9) Traumatic ICA occlusion caused by blunt injury is not uncommon in adults, but is rare in childhood. Here we describe a case of traumatic cervical ICA occlusion caused by extension and rotational injury in an infant.

\section{Case Report}

A 10-month-old boy fell to the ground from a baby carrier attached to a standing bicycle. His mother had parked the bicycle with the boy in the baby carrier attached to the bicycle, and left him unattended. Immediately after he fell to the ground, he cried out loud. On admission to another hospital,

Received August 4, 2004; Accepted May 20, 2005 physical examination found bruises on the scalp in the right temporal region and the right shoulder, but no wound in the neck. Chest radiography identified a right clavicular fracture and he underwent conservative therapy. No neurological deficit was noted. However, he began to vomit and became drowsy 19 hours after the trauma. Computed tomography (CT) detected cerebral infarct, so he was transferred to our service.

On admission, his level of consciousness was scored 9 on the pediatric coma scale. His pupils were equal in size, and reacted promptly to light. There was conjugate deviation of the eyes towards the right side. He also had left hemiparesis. No bruits were audible over the calvarium or in the neck on either side. Carotid pulsations in the neck were equal on both sides. Cranial radiography revealed a linear fracture running from the parietal to the occipital bone on the right. CT taken at approximately 24 hours after the trauma demonstrated a low density area in the territory of the right middle cerebral artery. Magnetic resonance imaging revealed a high signal intensity in the same area on both $\mathrm{T}_{2}$-weighted and diffusion-weighted images (Fig. 1). Ultrasonography of the neck revealed a hyperechoic lesion in the wall of the right ICA. Color flow Doppler sonography detected no blood flow within 

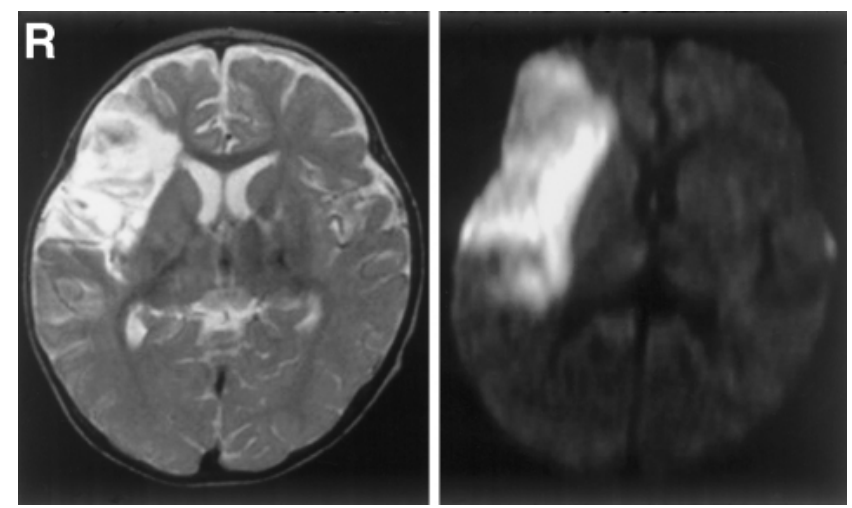

Fig. $1 \mathrm{~T}_{2^{-}}$(left) and diffusion-weighted (right) magnetic resonance images taken 26 hours after injury showing a hyperintense region in the right middle cerebral artery area, consistent with acute cerebral infarct.

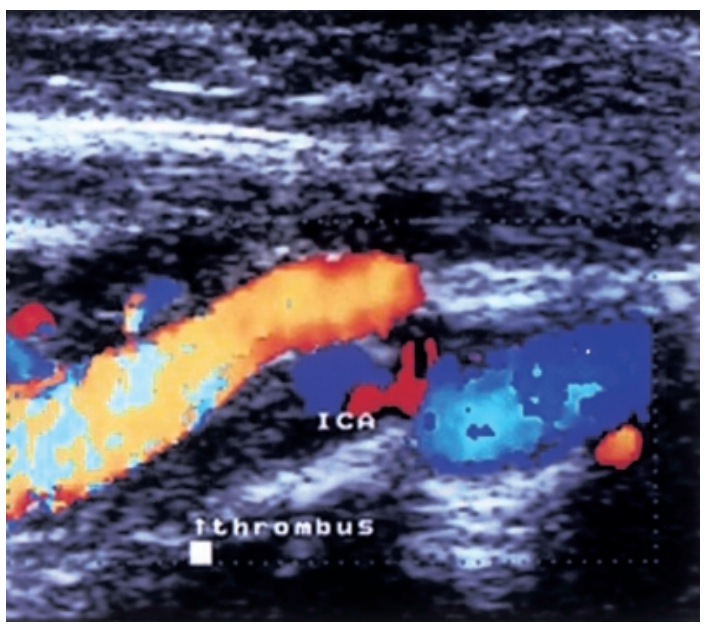

Fig. 2 Ultrasonogram of the neck 26 hours after trauma revealing a hyperechoic lesion in the wall of the right internal carotid artery (ICA), consistent with the absence of normal blood flow.

the right ICA (Fig. 2). Angiography disclosed complete occlusion of the right ICA $10 \mathrm{~mm}$ distal from the bifurcation of the common carotid artery (Fig. 3) and complete obliteration of the upper trunk of the right middle cerebral artery (Fig. 4).

The diagnosis was traumatic occlusion of the right cervical ICA and embolic obstruction of the upper trunk of the right middle cerebral artery. The bruising was located on the head and shoulder, but not on the neck, so the patient was considered to have sustained stretching of ICA associated with extension and rotational head injury. He underwent conservative therapy with hemodilution. His hemiparesis gradually improved and he was transferred to the rehabilitation service.

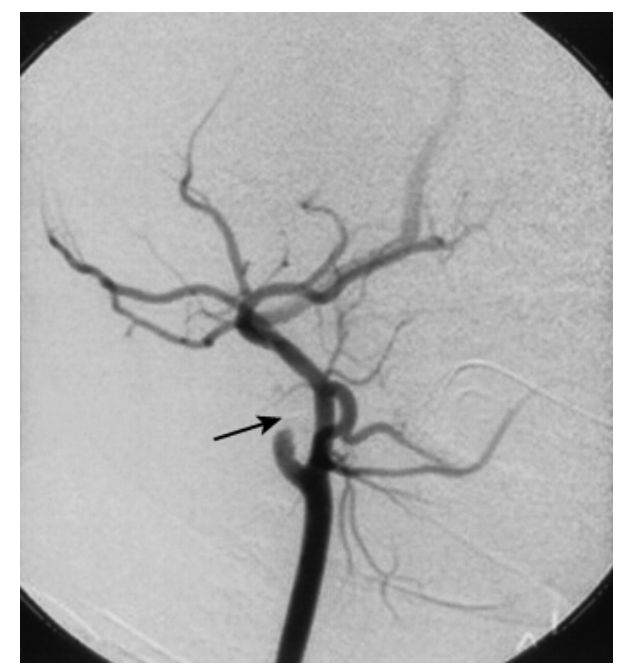

Fig. 3 Angiogram of the right common carotid artery (lateral view) demonstrating complete occlusion of the internal carotid artery (arrow), $10 \mathrm{~mm}$ distal from the bifurcation.

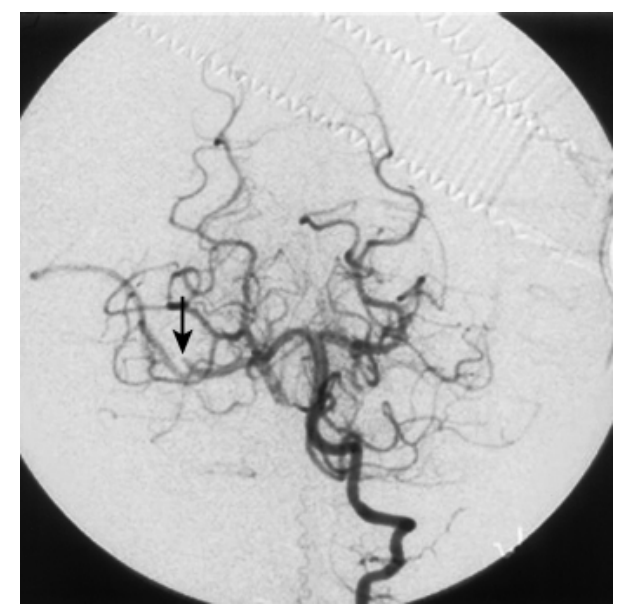

Fig. 4 Angiogram of the left vertebral artery (anteroposterior view) demonstrating good flow in the right middle cerebral artery via the posterior communicating artery, but the upper trunk (arrow) interrupted by embolus.

\section{Discussion}

Traumatic ICA occlusion caused by blunt injury is not uncommon in adults, but is rare in childhood. A review of the U.S. National Pediatric Trauma Registry found only 15 cases $(0.03 \%)$ of ICA occlusion caused by blunt injury among 57,659 patients with blunt trauma aged less than 19 years old.5) Traumatic ICA occlusion caused by blunt injury is extremely rare in infants aged less than 12 months. The only previously reported case occurred in an 11-month-old girl who fell to the floor. ${ }^{1)}$ Radiological 
examination revealed a linear fracture in the temporal to occipital bone and complete ICA occlusion at the supraclinoid portion. The previous and present cases indicate that traumatic ICA occlusion caused by blunt injury can occur in infants. However, the occlusions had very different locations in the extracranial cervical ICA in our case and the intracranial ICA in the previous case.

The causes of traumatic ICA occlusion can be classified into four groups ${ }^{3)}$ : Type 1 injury induced by a direct blow to the neck, type 2 injury induced by stretching of the ICA associated with neck hyperextension, type 3 injury associated with intraoral trauma, and type 4 injury associated with fractures at the skull base. Type 2 injury, as in our case, is considered to result from dissection or subintimal hemorrhage of the carotid wall. ${ }^{2,6)}$ Obstructions are often located at or just above the bifurcation of the common carotid artery in this type of traumatic ICA occlusion, in contrast to the ICA occlusion due to atherosclerotic change. ${ }^{4,11)}$

Traumatic ICA occlusion often leads to a poor outcome and the mortality is $37 \%$, regardless of the mechanism of occlusion. ${ }^{10,11)}$ Insufficient collateral circulation is the major cause of neurological deterioration. However, many patients may remain asymptomatic because they have sufficient collateral circulation, so the correct diagnosis is not established. Since collaterals from the posterior circulation frequently persist in infants, as in the present case, asymptomatic ICA occlusion may be more common in infants than in adults, suggesting that traumatic ICA occlusion in infants might tend to evade correct diagnosis. This inference suggests that traumatic ICA occlusion is not exceptionally rare in infants.

Traumatic ICA occlusion sometimes presents with delayed clinical onset, as in our case. Neurological deficits appear at more than 6 hours after trauma. ${ }^{2,4,6-8,11)}$ Delayed onset may be a consequence of progressive occlusion caused by dissection or subintimal hemorrhage. ${ }^{2,6)}$ Embolism is another cause of neurological deterioration after traumatic ICA occlusion, even in patients with sufficient collateral circulation. ${ }^{2,6,7)}$ Heparinization and anticoagulation therapy are recommended to prevent embolism after traumatic ICA occlusion. ${ }^{2)}$ Early diagnosis is probably critical for maximizing the effect of such therapy. ${ }^{10}$ ) The presence of chest trauma, including cervical fracture, may suggest the possibility of traumatic ICA occlusion in both adults and children. Investigation for traumatic ICA occlusion is of vital importance, even if there is no wound in the neck. ${ }^{1,5,7)}$ Ultrasonography is most useful for detecting traumatic ICA occlusion before it becomes symptomatic in an infant.

Extension and rotational head injury are likely if an infant falls from a baby carrier attached to a standing bicycle. Since such baby carriers on bicycles have become increasingly popular among young mothers in Japan, traumatic ICA occlusion may become more common in infants. The mother in the present case left the patient in the baby carrier of her bicycle unattended. We would like to emphasize that leaving a baby in a carrier on a standing bicycle is extremely hazardous and corresponds "Neglect" of parent's duties that is necessary to protect his or her baby.

\section{References}

1) Dharker SR, Dharker RS: Traumatic occlusion of internal carotid artery in an infant. Surg Neurol 9: 77-78, 1978

2) Elliott JP, Newell DW: Traumatic cerebrovascular injury, in Winn HR (ed): Youmans Neurological Surgery, ed 5. Philadelphia, PA Saunders, 2003, pp 5203-5221

3) Fleming JF, Petrie D: Traumatic thrombosis of the internal carotid artery with delayed hemiplegia. Can J Surg 11: 166-172, 1968

4) Hockaday TD: Traumatic thrombosis of the internal carotid artery. J Neurol Neurosurg Psychiatry 22: 229-231, 1959

5) Lew SM, Frumiento C, Wald SL: Pediatric blunt carotid injury: a review of the National Pediatric Trauma Registry. Pediatr Neurosurg 30: 239-244, 1999

6) Mokri B, Piepgras DG, Houser OW: Traumatic dissections of the extracranial internal carotid artery. J Neurosurg 68: 189-197, 1988

7) Olafson RA, Christoferson LA: The syndrome of carotid occlusion following minor craniocerebral trauma. J Neurosurg 33: 636-639, 1970

8) Schneider RC, Lemmen LJ: Traumatic internal carotid artery thrombosis secondary to nonpenetrating injuries to the neck; a problem in the differential diagnosis of craniocerebral trauma. J Neurosurg 9: 495-507, 1952

9) Verneuil M: Contusions multiples; delire violent; hemiplegie a droite, signes de compression cerebrale. Bull Acad Natl Med (Paris) 36: 46-56, 1872 (Fre)

10) Watridge CB, Muhlbauer MS, Lowery RD: Traumatic carotid artery dissection: diagnosis and treatment. J Neurosurg 71: 854-857, 1989

11) Yamada S, Kindt GW, Youmans JR: Carotid artery occlusion due to nonpenetrating injury. J Trauma 7: 333-342, 1967

Address reprint requests to: T. Igarashi, M.D., Department of Neurological Surgery, Nihon University School of Medicine, 30-1 Oyaguchi-kamimachi, Itabashi-ku, Tokyo 173-8610, Japan. e-mail: tkigsolm@med.nihon-u.ac.jp 